\title{
MicroRNA-143 targets ERK5 in granulopoiesis and predicts outcome of patients with acute myeloid leukemia
}

Jens-Uwe Hartmann', Daniela Bräuer-Hartmann', Miroslava Kardosova², Alexander A. Wurm, Franziska Wilke, Cindy Schödel ${ }^{1}$, Dennis Gerloff ${ }^{1}$, Christiane Katzerke ${ }^{1}$, Rosanna Krakowsky ${ }^{1}$, Carolina Yaeko Namasu ${ }^{1}$, Marius Bill', Sebastian Schwind', Carsten Müller-Tidow ${ }^{3}$, Dietger Niederwieser ${ }^{1}$, Meritxell Alberich-Jorda ${ }^{2}$ and Gerhard Behre ${ }^{1}$

\begin{abstract}
Hematopoiesis, the formation of blood cells from hematopoietic stem cells (HSC), is a highly regulated process. Since the discovery of microRNAs (miRNAs), several studies have shown their significant role in the regulation of the hematopoietic system. Impaired expression of miRNAs leads to disrupted cellular pathways and in particular causes loss of hematopoietic ability. Here, we report a previously unrecognized function of miR-143 in granulopoiesis. Hematopoietic cells undergoing granulocytic differentiation exhibited increased miR-143 expression. Overexpression or ablation of miR-143 expression resulted in accelerated granulocytic differentiation or block of differentiation, respectively. The absence of miR-143 in mice resulted in a reduced number of mature granulocytes in blood and bone marrow. Additionally, we observed an association of high miR-143 expression levels with a higher probability of survival in two different cohorts of patients with acute myeloid leukemia (AML). Overexpression of miR-143 in AML cells impaired cell growth, partially induced differentiation, and caused apoptosis. Argonaute2-RNAImmunoprecipitation assay revealed ERK5, a member of the MAPK-family, as a target of miR-143 in myeloid cells. Further, we observed an inverse correlation of miR-143 and ERK5 in primary AML patient samples, and in CD34 ${ }^{+}$HSPCS undergoing granulocytic differentiation and we confirmed functional relevance of ERK5 in myeloid cells. In conclusion, our data describe miR-143 as a relevant factor in granulocyte differentiation, whose expression may be useful as a prognostic and therapeutic factor in AML therapy.
\end{abstract}

\section{Introduction}

MicroRNAs (miRNAs) are a class of small non-coding RNAs (ncRNAs), 19-25 nucleotides in length, which can inhibit the translation or induce the destabilization and/or degradation of their mRNA targets, usually by binding in an incomplete manner to the $3^{\prime}$ untranslated region ( $3^{\prime}$ UTR) of their respective targets ${ }^{1}$. Since their initial discovery, miRNAs have been found to play

\footnotetext{
Correspondence: Gerhard Behre (Gerhard.Behre@medizin.uni-leipzig.de) ${ }^{1}$ Division of Hematology and Oncology, Leipzig University Hospital, Leipzig, Germany

${ }^{2}$ Institute of Molecular Genetics of the ASCR, v.v.i. Laboratory of Haematooncology, Prague, Czech Republic

Full list of author information is available at the end of the article. Edited by M Diederich
}

important roles in proliferation, differentiation, and apoptosis ${ }^{2-4}$. miRNAs have also been implicated in all stages of hematopoiesis including maintenance of hematopoietic stem cells (HSCs) and differentiation into mature effector cells ${ }^{5,6}$. We and others have shown that miRNAs play a key role as oncogenes ${ }^{7-9}$ or tumor suppressors $^{10-12}$ in leukemia, the malignant transformation of hematopoiesis.

Acute myeloid leukemia (AML) as a very aggressive leukemic subtype is characterized by a large genetic heterogeneity and the presence of immature abnormal myeloid progenitor cells in the bone marrow ${ }^{13}$. Despite improvements in diagnosis and therapy, the 5-year survival rate of adult AML patients is only 30\% (http://seer.cancer.gov).

\section{(c) The Author(s) 2018}

(c) (i) Open Access This article is licensed under a Creative Commons Attribution 4.0 International License, which permits use, sharing, adaptation, distribution and reproduction c. in any medium or format, as long as you give appropriate credit to the original author(s) and the source, provide a link to the Creative Commons license, and indicate if changes were made. The images or other third party material in this article are included in the article's Creative Commons license, unless indicated otherwise in a credit line to the material. If material is not included in the article's Creative Commons license and your intended use is not permitted by statutory regulation or exceeds the permitted use, you will need to obtain permission directly from the copyright holder. To view a copy of this license, visit http://creativecommons.org/licenses/by/4.0/. 
Diagnostic strategies continuously aim to identify novel prognostic markers such as gene mutations and DNA methylation to improve therapy options for patients ${ }^{14}$. In this context, abnormal expression of different miRNAs has been detected in distinct AML subtypes leading to activation or inhibition of essential pathways in leukemogenesis ${ }^{15}$. However, the function of individual miRNAs during normal and malignant hematopoiesis and their role as prognostic markers remains largely unknown.

miR-143 is an miRNA commonly seen to be downregulated in a variety of cancers, including hematopoietic malignancies ${ }^{16,17}$. Several studies implicate an important role of miRNA-143 to promote differentiation and to inhibit proliferation since it targets a number of cellular factors and pathways involved in transcription ${ }^{18-20}$. miR143 is shown to target several tumor-associated factors and thereby interfere with fundamental cellular processes often found deregulated in cancer ${ }^{21-23}$. Due to this, miR143 could have been described as tumor suppressor and prognostic marker in a wide range of tumors ${ }^{24-26}$.

ERK5 (extracellular signal-regulated kinase 5; MAPK7; mitogen-activated protein kinase 7) as a part of the MEK/ ERK-pathway ${ }^{27}$ is a verified miR-143 target in solid cancers $^{28-30}$. The transcription factor ERK5 is a central mediator of cell survival, proliferation, differentiation, and apoptotic regulation of normal cells ${ }^{31-33}$. Deregulation and activation of ERK5 has been shown to be a frequent event in the onset and progression of cancer ${ }^{34-36}$. Furthermore, recent publications describe the involvement of ERK5 in therapy response, including leukemia ${ }^{37,38}$. The interaction between the tumor suppressor miR-143 and oncogenic ERK5 signaling is well characterized in solid cancers, but their interplay is rather unknown in the background of AML.

In the present study, we explore the role of miR-143 in hematopoietic differentiation and AML. We found miR143 to be upregulated during granulocytic differentiation of primary human $\mathrm{CD} 34^{+}$stem/progenitor cells (HSPCs), primary acute promyelocytic leukemia (APL) patient samples, and various AML cell lines. Furthermore, we demonstrate the importance of miR-143 expression for granulocytic differentiation in vitro and in vivo. In line with this, we identified high miR-143 expression as a favorable prognostic factor in AML. By ectopic expression of miR-143, we showed ERK5, an important member of the MAP-kinase pathway, as a target of miR-143. This finding is supported by inverse correlation of miR-143 expression and ERK5 protein in CD34 ${ }^{+}$HSPCs and AML patient samples. Taken together, our data depict an important role of miR-143 in normal granulocytic differentiation and treatment response in AML and thereby provides a novel source for clinical applications of miRNAs in the context of myeloid leukemia.

\section{Results}

miR-143 is upregulated during granulopoiesis in vitro and in vivo

Even though several miRNAs have been shown to be regulated in granulopoiesis, whether miRNAs are downstream targets of cytokines and how this regulation is instrumental in granulopoiesis is not known. In order to identify differentially expressed miRNAs during granulocytic differentiation, we treated primary human $\mathrm{CD} 34^{+}$ HSPCs with G-CSF or vehicle $\left(\mathrm{H}_{2} \mathrm{O}\right)$. Next-generation sequencing (NGS) was performed at day 7 of treatment and revealed several differentially regulated miRNAs, including miR-143 as the most upregulated (3.96-fold); (Fig. 1a). Differentiation status and viability was assessed by FACS-based measurement of CD15 and morphology (Supplementary Fig. 1a-c). To confirm NGS data, miR143 expression was measured in G-CSF treated CD34 ${ }^{+}$ HSPCs by qPCR. We observed a continuous increase of miR-143 levels until day 14 followed by a decline thereafter (Fig. 1b). To exclude cell-specific effects, we analyzed miR-143 expression in different hematopoietic cell lines (U937, NB4, and K562-C/EBP $\alpha$-ER) undergoing granulocytic differentiation. All cells showed a significant upregulation of miR-143 expression at the indicated time points compared to the control (Fig. 1c). Granulocytic differentiation was confirmed by morphology and FACSbased measurement of CD11b (Supplementary Fig. 1d-f). Since ATRA causes granulocytic differentiation, we also analyzed bone marrow and blood samples from APLpatients undergoing ATRA-based therapy. qPCR analysis revealed the ATRA-dependent upregulation of miR-143 after treatment (Fig. 1d, Supplementary Table 1).

\section{miR-143 is important for granulocytic differentiation}

To address the role of miR-143 in hematopoietic differentiation, we performed stable overexpression $(\mathrm{O} / \mathrm{E})$ or knockdown $(\mathrm{kd})$ of miR-143 in HSPCs and hematopoietic cell lines. Overexpression of miR-143 in CD34 ${ }^{+}$HSPCs followed by G-CSF treatment resulted in accelerated expression of CD15 at day 7 (Fig. 2a), whereas the knockdown of miR-143 caused a decrease in CD15 expression at day 11 (Fig. 2b). Transient overexpression of miR-143 in the myeloid cell line K562-C/EBP $\alpha$-ER followed by induction of granulocytic differentiation with $\beta$-estradiol also caused an accelerated differentiation compared to control cells (Fig. 2c, Supplementary Fig. 2a). In contrast to this, lentiviral reduction of miR-143 levels in $\mathrm{K} 562-\mathrm{C} / \mathrm{EBP} \alpha-\mathrm{ER}$ cells resulted in a reduced CD11b expression upon $\beta$-estradiol treatment compared to the control (Fig. 2d, Supplementary Fig. 2b). To confirm these data, we performed knockdown of miR-143 in NB4 and U937 cells and observed comparable results (Supplementary Fig. 2c, d). 

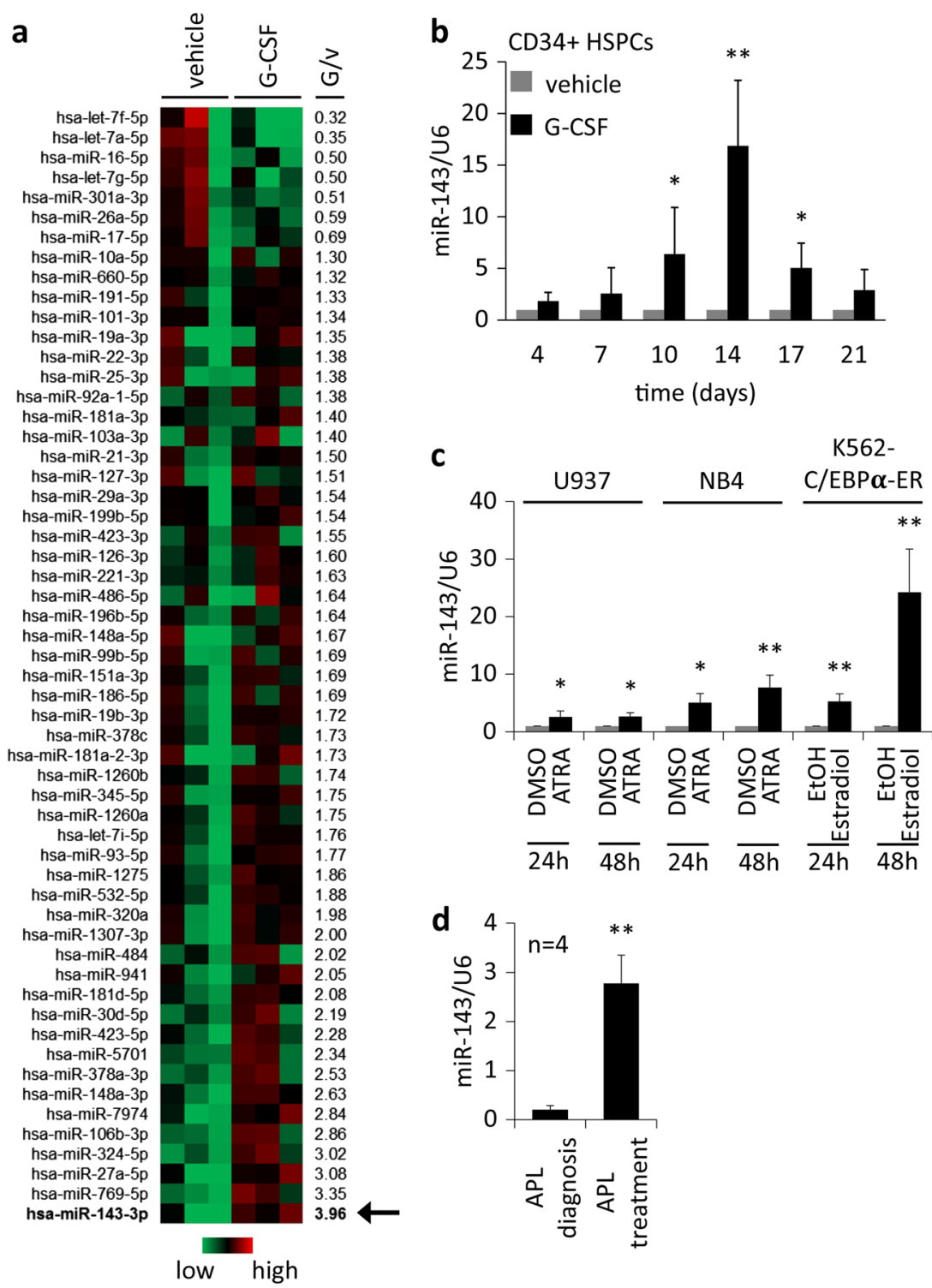

Fig. 1 miR-143 is upregulated during granulopoiesis in vitro and in vivo. a Heat map of differentially regulated microRNAs in G-CSF or vehicletreated human $\mathrm{CD}_{3} 4^{+}$hematopoietic stem cells (HSPCs). miRNA expression pattern was evaluated by next-generation sequencing (NGS), 7 days after treatment. The miRNA expression was calculated to the median expression of each miRNA. Numbers beside the heat map represent the ratio G-CSF/ vehicle from all three experiments. miR-143 expression is marked with an arrow (red $=$ high, green $=$ low). $\mathbf{b}$ qPCR analysis of miRNA-143 expression in G-CSF or vehicle $\left(\mathrm{H}_{2} \mathrm{O}\right)$ treated CD34 $4^{+}$HSPCs over 21 days. c ATRA-treated NB4 and U937 cells as well as $\beta$-estradiol treated K562-C/EBPa-ER at indicated time points. Bars represent the mean \pm SD from at least three independent experiments $(\mathbf{b}, \mathbf{c})$. $\mathbf{d}$ qPCR analysis of miR-143 expression in primary APL patient bone marrow or peripheral blood samples at diagnosis and during ATRA treatment. Bars represent the mean \pm SD $[n=4]$. * $P \leq$ $0.05 ;{ }^{* *} P \leq 0.01$, unpaired two-tailed $t$-tests

\section{Stable knockdown of miR-143 leads to a reduction of granulocytic cells in mice}

To investigate miR-143 expression during normal granulopoiesis, we sorted distinct murine bone marrow populations from $\mathrm{C} 57 \mathrm{BL} / 6 \mathrm{NCrl}$ mice. qPCR analysis showed a steady increase of miR-143 expression according to the differentiation progress, with the highest miR143 expression levels in mature granulocytes (Fig. 3a).
Next, we investigated whether downregulation of miR143 would impair granulocytic differentiation in vivo. Therefore, we performed murine bone marrow transplantation experiments with manipulated lineage ${ }^{-} \mathrm{Sca}-1^{+}$ $\mathrm{cKit}^{+}$(LSK) cells, population enriched for hematopoietic stem and progenitor cells (Fig. 3b). Sorted LSK cellsisolated from C57BL/6NCrl mice (Ly5.2) were transduced with pseudoviral particles carrying lentivector 


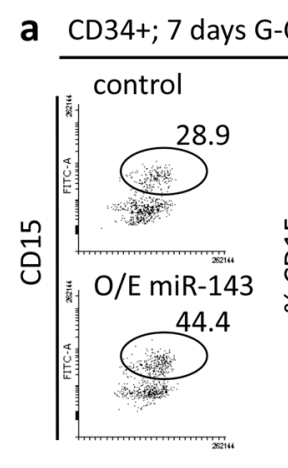

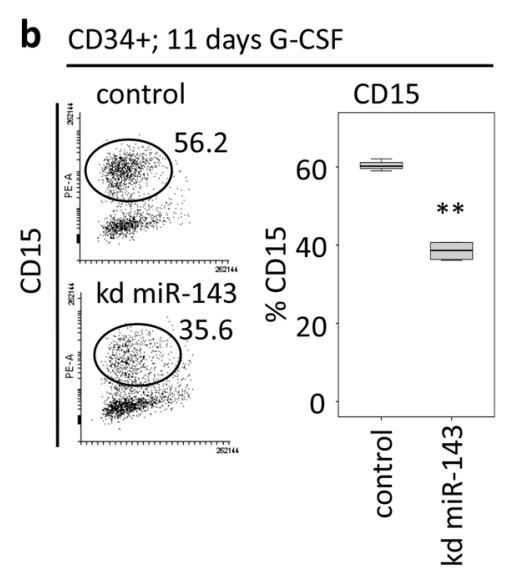
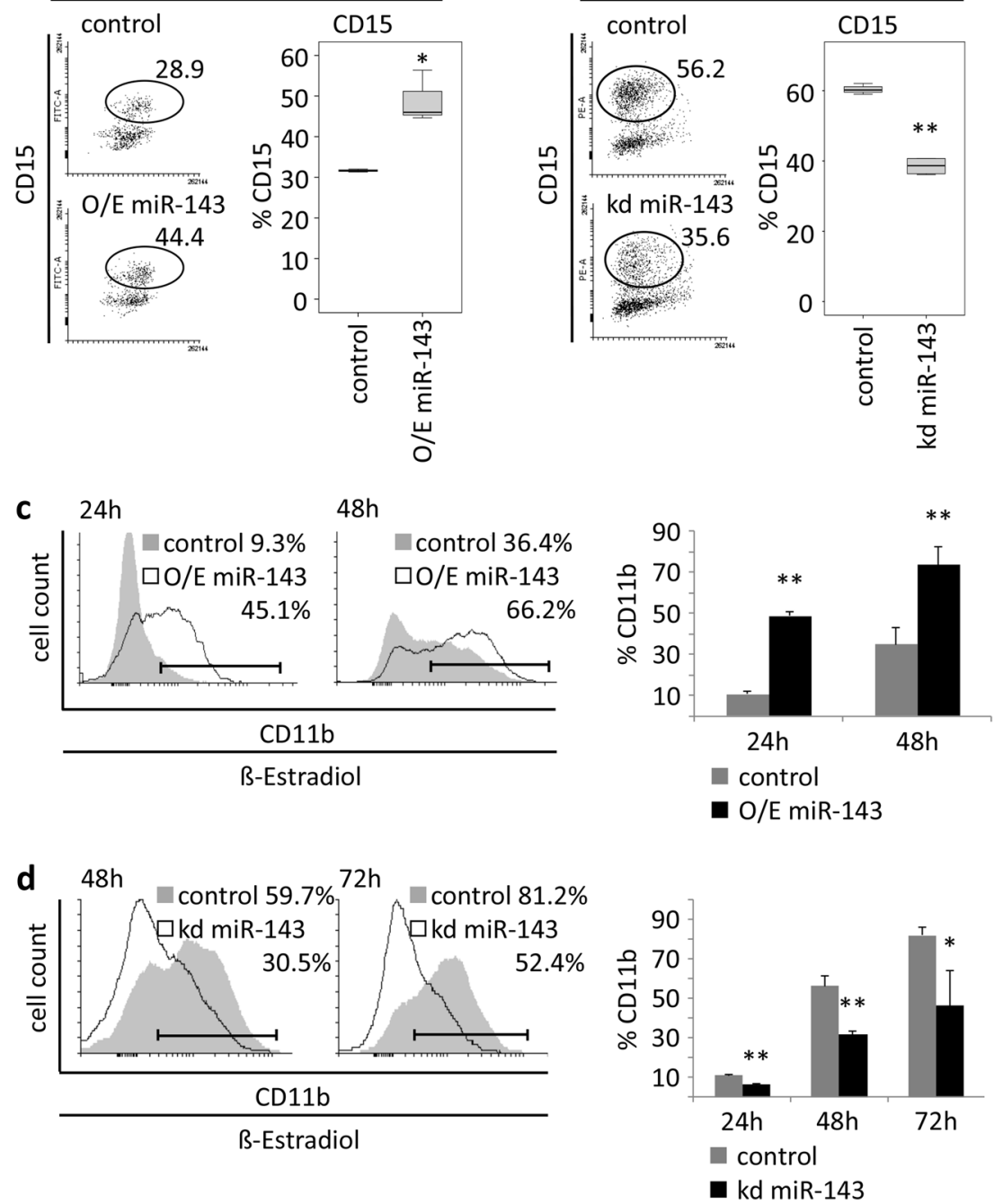

Fig. 2 miR-143 is necessary for granulocytic differentiation. $\mathbf{a}$, b FACS analysis of CD15 expression of G-CSF-treated CD34 ${ }^{+}$HSPCs (a) 7 days after stable overexpression (b) 11 days after stable knockdown of miR-143. c, d FACS analysis of CD11b expression at indicated time points in K562-C/ EBPa-ER cells (c) after transient miR-143 overexpression or (d) stable miR-143 knockdown and treatment with $\beta$-estradiol. Numbers in dot blots indicate percentage of $\mathrm{CD}_{15} 5^{+}$or $\mathrm{CD} 11 \mathrm{~b}^{+}$cells. Bars in diagrams represent the mean \pm SD from three independent experiments. $P$ values are relative to control. ${ }^{*} P \leq 0.05 ;{ }^{* *} P \leq 0.01$, unpaired two-tailed $t$-tests

for miR-143 knockdown (miRZip-143) or control vector (miRZip-control). Importantly, miRZip vector contains a GFP reporter that allows tracking of infected cells. Since we did not sort $\mathrm{GFP}^{+}$cells prior to transplantation, the donor-derived Ly5.2 population consists of a mixture of transduced $\left(\mathrm{GFP}^{+}\right)$and untransduced $\left(\mathrm{GFP}^{-}\right)$cells. Efficiency of transduction was similar in both groups as measured by assessing GFP positivity $48 \mathrm{~h}$ post transduction (data not shown). Recipient mice were killed and blood and bone marrow were analyzed 3 weeks after transplantation. As expected, we did not observe changes in the engraftment of total Ly5.2 $2^{+}$cells or contribution of $\mathrm{GFP}^{+}$donor cells to the hematopoietic system of the recipient mice (Supplementary Fig. 3a, b). However, flow cytometry analysis showed a significant reduction of $\mathrm{CD}_{11 \mathrm{~b}}{ }^{+} / \mathrm{Ly}_{6 \mathrm{G}}{ }^{+}$mature granulocytes in the $\mathrm{GFP}^{+}$fraction of the cells with miR-143 knockdown (kd miR-143) compared to $\mathrm{GFP}^{+}$control cells both in peripheral blood and bone marrow of recipient mice (Fig. 3c). No effect was observed in the $\mathrm{GFP}^{-}$non-infected fraction. Of note, the percentage of mature $\mathrm{CD}_{11} \mathrm{~b}^{+} / \mathrm{Ly} 6 \mathrm{C}^{+}$monocytes were not affected (Supplementary Fig. 3c). To determine cell morphology of the donor-derived cells, we sorted Ly5.2 $2^{+} / \mathrm{GFP}^{+}$cells from BM of recipient mice, cytospun them, and performed May-Grünwald/Giemsa staining. In line with the flow cytometric data, we observed reduced 
a

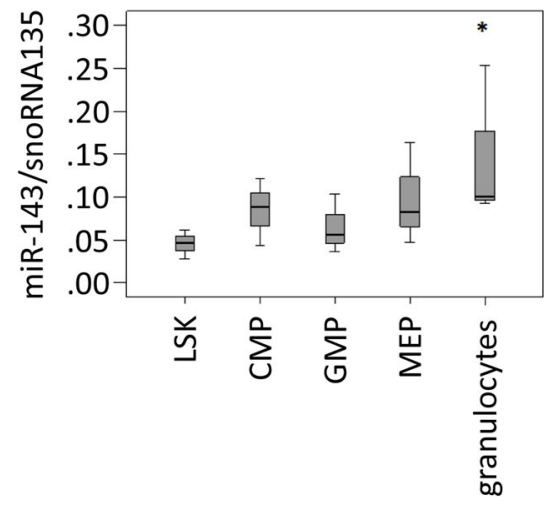

b

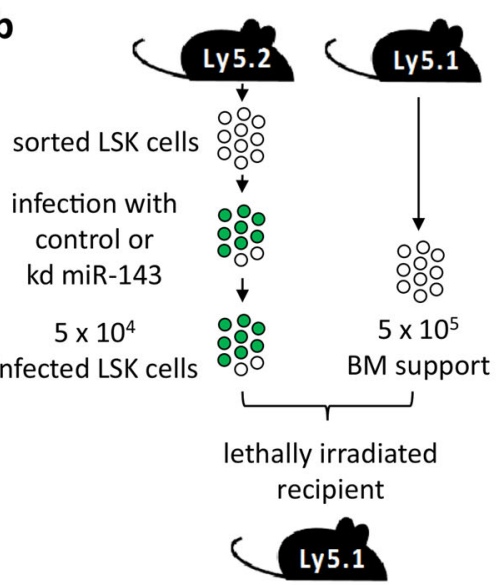

$B L$

control kd miR-143

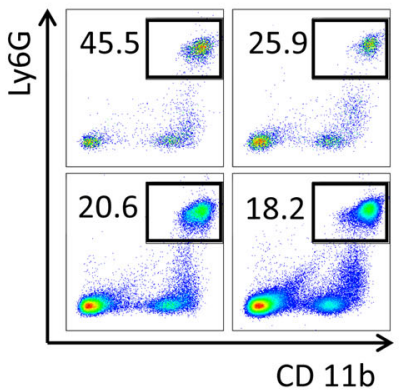

d

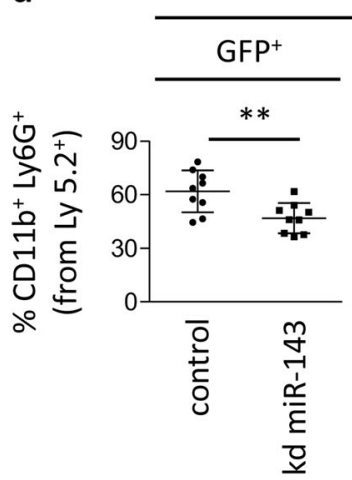

BM

control kd miR-143

$\mathrm{GFP}^{+}$

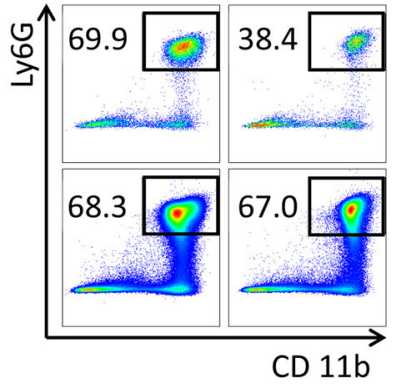

BM

$\mathrm{GFP}^{-}$

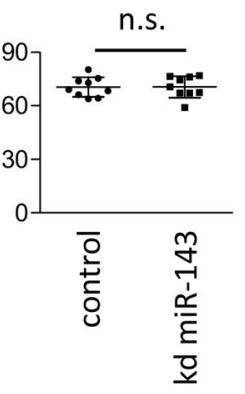

BL

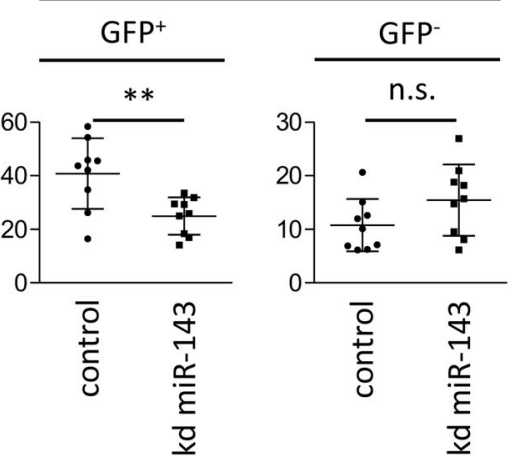

Fig. 3 Stable knockdown of miR-143 leads to reduced granulocytic differentiation in vivo. a qPCR for miR-143 expression in sorted mouse bone marrow cell subpopulations. Bars represent the mean \pm SD from at least three independent cell sortings. Bars are represented as $\Delta C t$ values. $P$ value of granulocytes is relative to all other analyzed samples. ${ }^{*} P<0.05$. b Schematic overview of the LSK-based knockdown (kd) transplantation model. LSK cells sorted from C57BI/6 Ly5.2 ${ }^{+}$mice were transduced with miRZip-143 (kd miR-143) or miRZip-control (control) lentivector-based constructs. Of note, miRZip construct contains a GFP reporter. Next, non-sorted LSK cells (containing a mixture of infected and non-infected cells) were mixed with $5 \times 10^{5}$ total bone marrow cells isolated from C57BI/6 Ly5.1 $1^{+}$congenic mice and transplanted into lethally irradiated C57BI/6 Ly5.1 ${ }^{+}$ recipients by tail vein injection. Importantly, the infection efficiency was similar in miRZip-143 and miRZip-control. Analysis of recipient mice was performed 3 weeks after transplantation. c Flow cytometric analysis representative plots from BM and BL of recipient mice transplanted with control (left panels) or miR143 knockdown (kd miR-143, right panels) LSK cells. Plots were generated by gating on donor-derived Ly5.2 $2^{+}$cells, and subsequently divided into GFP (infected) and GFP ${ }^{-}$(non-infected) cells. Black boxes indicate percentage of mature Ly6G ${ }^{+} / \mathrm{CD}^{+} 11 \mathrm{~b}^{+}$granulocytes. The percentage of donor-derived mature granulocytes in BM and BL of recipient mice were reduced when miR-143 levels were downregulated (GFP+ $\mathrm{kd}$ miR-143) in comparison to control (GFP+ control). GFP- fraction (lower panels) served as an additional negative control. Dead cells were excluded from analysis by Hoechst 33258 staining. d Quantification of the flow cytometry analysis described in panel $\mathbf{c}$. $Y$-axis indicates the percentage of mature $\mathrm{Ly}_{6 \mathrm{G}^{+}} / \mathrm{CD} 11 \mathrm{~b}^{+}$granulocytes derived from donor Ly5.2 $2^{+}$cells. Bars represent the mean \pm SD from two independent experiments, $n=9$ (control) or 10 (kd miR-143) mice, ${ }^{* *} P<0.01$, unpaired two-tailed $t$-tests were used to assess statistical significance. LSK, Lin ${ }^{-}$Sca- $1^{+}$ $\mathrm{CKit}^{+}$cells; CMP, common myeloid progenitors; GMP, granulocyte-macrophage progenitors; MEP, megakaryocyte erythroid progenitors and granulocytes; Kd, knockdown; BM, bone marrow; BL, blood 
numbers of mature neutrophils in mice transplanted with miR-143 knockdown cells compared to the controls (Supplementary Fig. 3d, e). Altogether, we demonstrated that miR-143 is gradually expressed during granulocytic differentiation in all developmental stages, detected the highest levels of miR-143 in mature granulocytes, and showed that miR-143 knockdown in LSK cells partially blocks granulocytic differentiation in vivo.

\section{High miR-143 expression is a prognostic factor for favorable outcome in AML}

To investigate the impact of miR-143 on AML prognosis, we analyzed miR-143 expression in the TCGA AML patient data set (GDC Data Portal). AML patients with high miR-143 expression showed a significantly longer overall survival (OS) than AML patients with low miR-143 expression. According to this, AML patients with high miR-143 expression had significantly lower percentages of blasts in peripheral blood and bone marrow, as well as significantly lower WBC counts than AML patients with low miR-143 expression (Fig. 4a, Supplementary Table 2). To verify our data, we analyzed miR143 expression in a second cohort, capturing $47 \mathrm{AML}$ patients, who received non-myeloablative (NMA) conditioning before allogeneic HSC transplantation. In this cohort, high miR-143 expression was associated with a significantly lower WBC count and lower percentages of blasts in peripheral blood, but not in bone marrow at diagnosis (Supplementary Fig. 4a). Whereas OS was not different in this patient cohort, AML patients with high miR-143 expression showed a significantly longer eventfree survival than patients with low miR-143 expression (Supplementary Fig. 4b). Further, we investigated miR143 expression in different cytogenetic AML subclasses of the TCGA data set and observed a significantly higher miR-143 expression in the MRC (British Medical Research Council) classified favorable risk group compared to the intermediate-risk and poor-risk group (Fig. 4b). Here, AML patients with an FLT3 receptor mutation, a mutation connected to unfavorable prognosis $^{39}$, showed the lowest miR-143 expression (Fig. 4b, c, Supplementary Table 2). qPCR analysis of a second AML patient set also showed that AML patients with an FLT3ITD mutation showed significantly lower miR-143 expression levels compared to FLT3 wild type (Fig. 4d, Supplementary Table 3).

\section{miR-143 impairs growth, survival, as well as partial differentiation of leukemic cells}

To investigate miR-143 effects in leukemic cells, we overexpressed miR-143 in 32D cells stably expressing FLT3-ITD. miR-143 enrichment was confirmed by qPCR (Fig. 5a). Cell growth analysis showed that 32D-FLT3-ITD cells overexpressing miR-143 showed significantly less cell growth than the control cells (Fig. 5b). Furthermore, miR143 overexpression resulted in a slight increase of the granulocytic surface marker Ly6G and a more mature morphology toward granulocytes (Fig. 5c, Supplementary Fig. 5). Additionally, we observed a significant increase in apoptotic cell number in miR-143 overexpressing 32DFLT3-ITD compared to the control (Fig. 5d). Furthermore, the inhibition of FLT3 signaling by the potent FLT3 inhibitor AC220 (Quizartinib) leads to the induction of miR-143 expression in 32D-FLT3-ITD cells $24 \mathrm{~h}$ after treatment (Fig. 5e). In turn, stable inhibition of miR-143 in 32D-FLT3-ITD cells partially prevents AC220mediated effects on apoptosis (Fig. 5f). To evaluate FLT3-ITD-independent effects of miR-143 on AML cells, we stably overexpressed miR-143 in freshly isolated AML patient bone marrow samples (Supplementary Table 4). FACS-based measurement of Annexin V-stained cells showed a significant increase in apoptosis in miR143 overexpressing cells compared to control cells (Fig. 5g). Finally, we also investigated miR-143 expression in bone marrow samples from AML patients receiving a 5azacytidine-based therapy. qPCR analysis showed that patients who respond to the therapy had significantly higher miR-143 levels at diagnosis than patients who did not respond (Fig. 5h, Supplementary Table 5).

\section{ERK5 is a target of miR-143}

Because miRNAs exert their function via targeting specific mRNAs, we used the argonaute 2-RNAimmunoprecipitation (AGO-RIP) technology to identify miR-143 target mRNAs in myeloid cells. miR-143 was stably overexpressed in $\mathrm{K} 562-\mathrm{C} / \mathrm{EBP} \alpha-\mathrm{ER}$ cells and AGORIP followed by NGS for mRNAs was performed (Supplementary Fig. 6a). We identified a total number of 635 mRNAs, which showed at least 10-fold increased binding to the argonaute 2 protein in the miR-143 overexpressing cells compared to the control cells (Fig. 6a, left panel). Using KEGG pathway analysis, we identified several pathways targeted by miR-143, including the MAPkinase-signaling pathway as the most targeted pathway (Fig. 6a, right panel). The transcription factor extracellular signal-regulated kinase 5 (ERK5), also known as mitogenactivated protein kinase 7 (MAPK7), is a main component of the MAPK-pathway ${ }^{27}$ and was significantly enriched in the AGO-RIP NGS screen (data not shown). The ERK5 3' UTR carries one conserved miR-143 binding site (Supplementary Fig. 6b). By semiquantitative PCR, we confirmed the NGS data and observed the enrichment of ERK5 mRNA in the AGO-RIP samples from miR-143 overexpressing cells compared to control cells and IgG antibody control (Fig. 6b). To prove the direct binding of miR-143 to the predicted binding site in the 3' UTR of ERK5 (Supplementary Fig. 6b), we performed a luciferase assay. Therefore, we co-transfected pGL3-constructs 

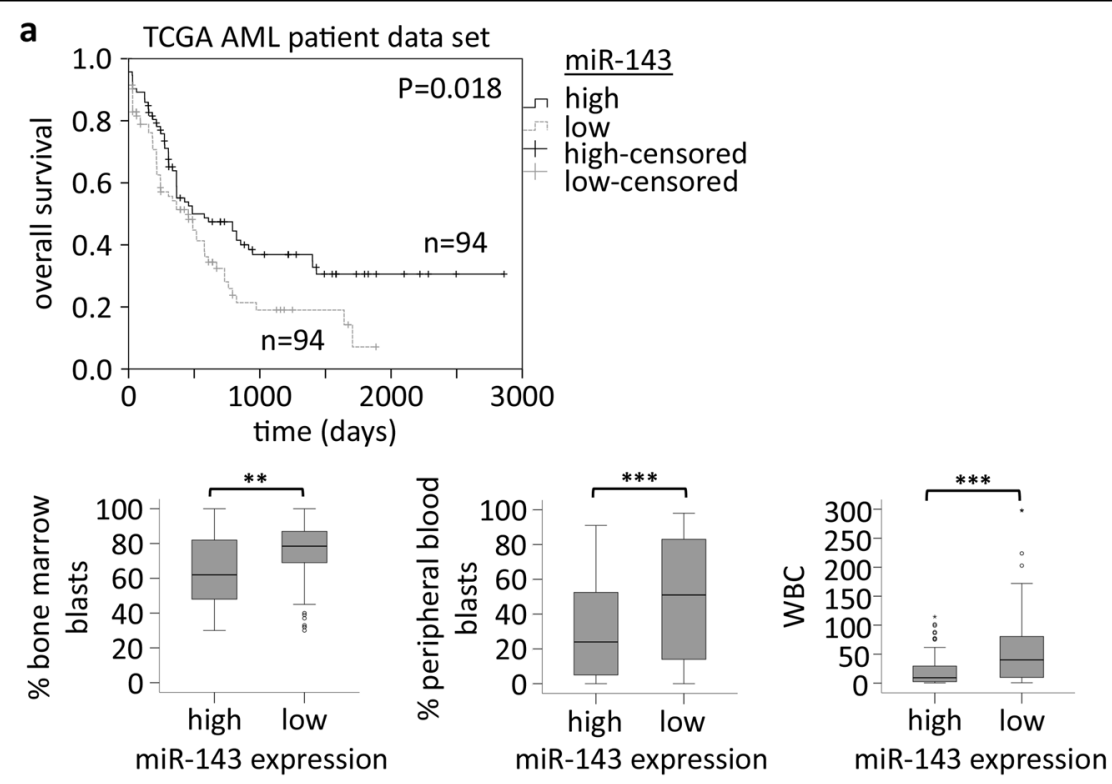

b

C TCGA AML patient data set
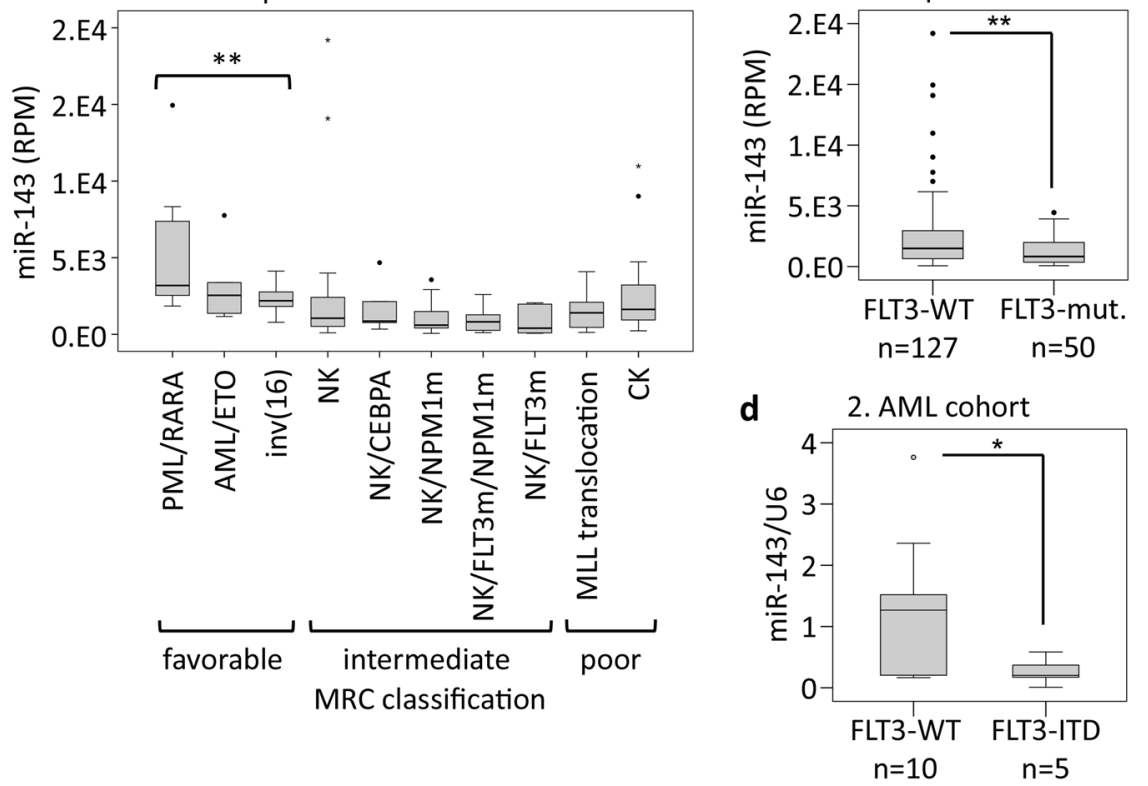

Fig. 4 miR-143 is a good prognostic factor in AML. a Overall survival of AML patients (TCGA Research Network), according to the dichotomized miR-143 expression; ( $n=188$; Mann-Whitney- $U$ test); (upper panel). Bone marrow blast count (BM blasts; left), peripheral blood blast count (PB blasts; middle), and number of white blood cells (WBC, right) in AML patients (TCGA Research Network), according to the dichotomized miR-143 expression (lower panel). Bars represent the median \pm SD [high miR-143, $n=94$; low miR-143, $n=94$ ]. ${ }^{* *} P<0.01$; ${ }^{* *} P<0.001$, unpaired two-tailed $t$-tests. b miR143 expression in different AML subgroups classified according to the MRC (the British Medical Research Council); (TCGA). c miR-143 expression in AML patients with and without FLT3-mutations; (TCGA). Bars represent the median \pm SD [FLT3-WT, $n=127$; FLT3-mut. $n=50]$, $P$ values are relative to FLT3-WT. ${ }^{* *} P<0.01$, unpaired two-tailed t-tests. d miR-143 expression in AML patients with wildtype FLT3 or a FLT3-ITD mutation (2. AML cohort). Bars represent the median \pm SD [FLT3-WT, $n=10$; FLT3-ITD, $n=5], P$ values are relative to FLT3-WT. ${ }^{*} P<0.05$, unpaired two-tailed $t$-tests

containing either the wild type ERK5-3' UTR, the ERK5-3' UTR with mutated miR-143 binding site or control (a shorten part of the C/EBP $\alpha-3^{\prime}$ UTR without any predicted miR-143 binding site) ${ }^{9}$ in combination with
miR-143 mimics or control oligos as well as a pRL construct. Luciferase activity measurement revealed the direct binding of miR-143 to the predicted binding site (Fig. 6c). In addition, Western blot analysis showed 

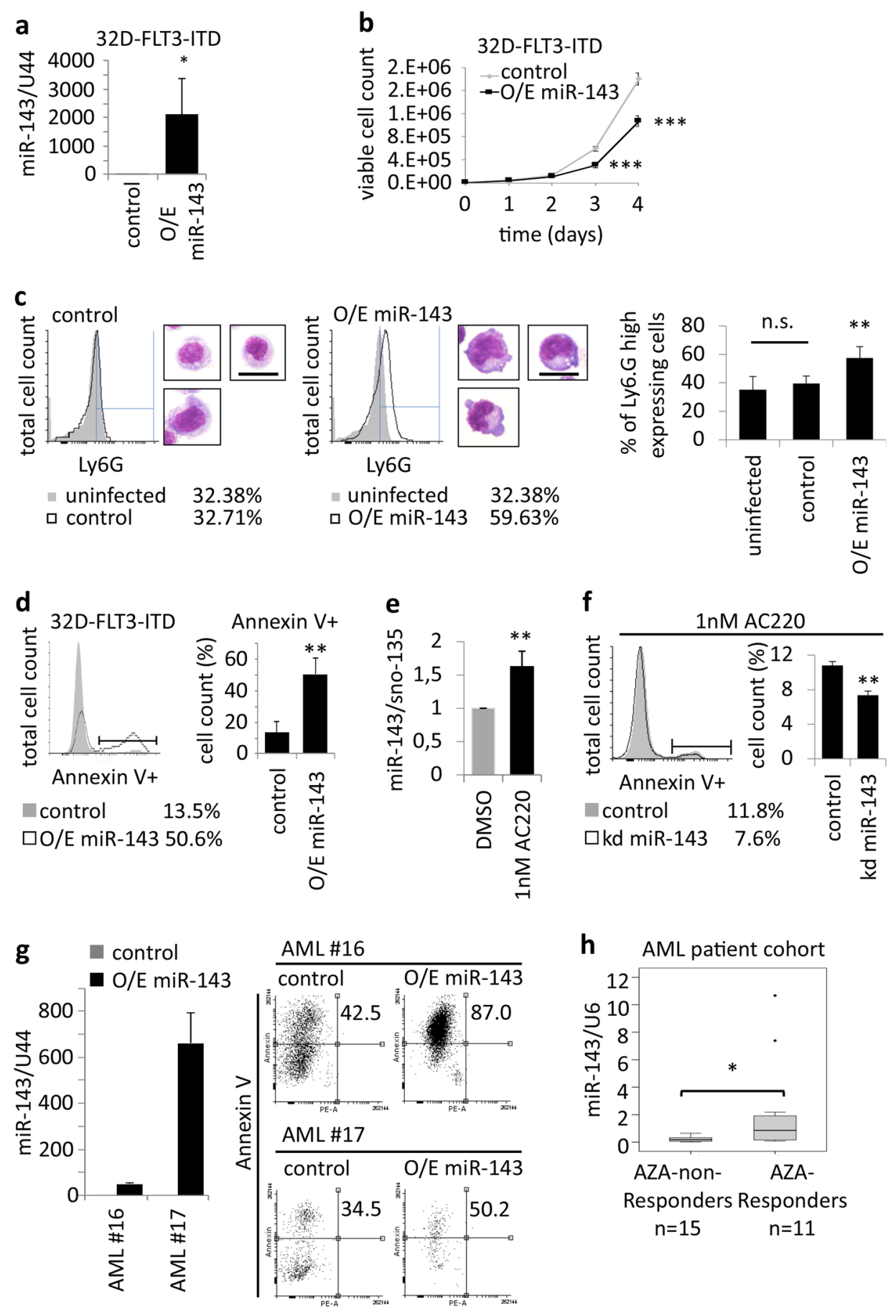

Fig. 5 (See legend on next page.)

miR-143-dependent changes in the protein levels of ERK5, activated ERK5, and the ERK5-downstream target c-Myc in K562-C/EBP $\alpha-E R$ and NB4 cells (Fig. 6d, Supplementary Fig. 6c, d).
ERK5 protein inversely correlates with miR-143 expression, interferes with granulocytic differentiation, and is relevant for myeloid cell survival

miR-143 expression and ERK5 protein levels were investigated during normal granulopoiesis of G-CSF-treated 
(see figure on previous page)

Fig. 5 miR-143 impairs AML cell growth, survival, and in partial differentiation. a qPCR analysis of exogenous miR-143 expression in 32D-FLT3ITD cells. Bars represent the mean \pm SD from three independent experiments. $P$ values are relative to control U44. ${ }^{*} P<0.05 ;{ }^{* *} P<0.01$, unpaired twotailed t-tests. b Cell growth curve of 32D-FLT3-ITD cells stably overexpressing miR-143 or control. Values represent mean \pm SD $[n=4]$. ${ }^{* * *} P<0.001$, unpaired two-tailed $t$-tests. c FACS analysis for Ly6G expression and representative morphology pictures of 32D-FLT3-ITD cells overexpressing miR143 or control. Scale bar $=10 \mu \mathrm{m}$. The column chart (right) represents the mean of three independent experiments \pm SD. $P$ values are relative to control. ${ }^{*} P \leq 0.01$, unpaired two-tailed $t$-tests. $\mathbf{d}$ FACS analysis for Annexin V-stained 32D-FLT-ITD cells overexpressing miR-143. Bars represent the mean \pm SD from three independent experiments. $P$ values are relative to control. ${ }^{*} P<0.01$, unpaired two-tailed $t$-tests. e qPCR for miR-143 expression in 32D-FLT3-ITD cells treated with $1 \mathrm{nM} \mathrm{AC220}$ for $24 \mathrm{~h}$. Bars represent the mean \pm SD from three independent experiments. $P$ values are relative to control. ${ }^{*} P<0.01$, unpaired two-tailed $t$-tests. $\mathbf{f}$ FACS analysis for Annexin V-stained 32D-FLT-ITD cells with stable knockdown of miR-143 or control after $24 \mathrm{~h}$ treatment with $1 \mathrm{nM} \mathrm{AC220}$. Bars represent the mean \pm SD from three independent experiments. $P$ values are relative to control. ${ }^{*} P<0.01$, unpaired two-tailed $t$-tests. $\mathbf{g}$ qPCR analysis of exogenous miR-143 expression in bone marrow cells of AML patients (left). Bars represented as technical replicates. FACS analysis of Annexin V-stained AML patient samples overexpressing miR-143 or control (right). Numbers in dot blots indicate the percentage of Annexin V-positive cells. $\mathbf{h}$ qPCR for miR-143 expression in AML patients undergoing 5-acazytidine therapy (AZA). Values represent the mean \pm SD [non-responder, $n=15$; responder, $n=11$ ]. $P$ values are relative to AZA-non-responders. ${ }^{*} P<0.05$, unpaired two-tailed $t$-tests

human $\mathrm{CD} 34^{+}$HSPCs and chemokine-induced granulopoiesis of K562-C/EBP $\alpha-E R, ~ N B 4$, and U937 cells. Western blot analysis of ERK5 protein in G-CSF-treated CD34 ${ }^{+}$HSPCs showed a decline in ERK5 protein amount over a time period of 17 days followed by an increase at later time points, which inversely correlated with the expression of miR-143 at the investigated time points (Figs. 1b, 7a). In ATRA-treated NB4 and U937 cells as well as $\beta$-estradiol-treated K562-C/EBP $\alpha$-ER cells we also observed an inverse correlation of ERK5 protein and miR143 expression (Fig. 1c; Fig. 7b, Supplementary Fig. 7a). Furthermore, analysis of AML patient samples revealed an inverse correlation of miR-143 and ERK5 protein in malignant cells (Fig. 7c; Supplementary Table 6). Finally, transient overexpression of ERK5 in K562-C/EBP $\alpha$-ER cells resulted in a decreased number of CD11b-positive cells and an increased number of apoptotic cells after $\beta$ estradiol-induced granulopoiesis (Fig. 7d, e; Supplementary Fig. 7b).

\section{Discussion}

miRNAs, a class of regulatory molecules, have been frequently found to be dysregulated in human cancers ${ }^{40}$. Mature miRNAs act as negative gene regulators and have been shown to function both as tumor suppressors and oncogenes ${ }^{41}$. Recent studies showed that miR-143 is involved in a wide range of differentiation processes ${ }^{21,42,43}$ and carries out tumor suppressive functions in several cancer types ${ }^{44-48}$. However, at the moment only little is known about the role of miR-143 in myeloid differentiation and AML.

In this study, we found a strong induction of miR-143 expression during granulocytic differentiation in cell lines, primary $\mathrm{CD} 34^{+}$HSPCs as well as APL patients treated with ATRA, which could be supported by publications from Donahue et al. and Batliner et $\mathrm{al}^{43,49}$. Furthermore, we could show that miR-143 expression reached the highest levels in mature granulocytes and knockdown of miR-143 reduced the number of mature granulocytes in vivo. Our overexpression and knockdown experiments clearly demonstrate the impact of miR-143 during granulopoiesis. Our observations indicate a functional relevance of miR-143 in later stages of granulocytic differentiation. This is in contrast to previous findings, which describe miR-143 in early differentiation processes ${ }^{50,51}$. In solid tumors miR-143 is well described, but only a few studies regard the role of miR143 in hematological malignancies ${ }^{52-54}$. In this study, we could show that overexpression of miR-143 leads to reduced proliferation and increased apoptosis of AML cells. Our observations are supported by data from Shen et al., which show that overexpression of miR-143 induces apoptosis in CML cells ${ }^{55}$. These data substantiate the tumor-suppressive function of miR-143 in the background of leukemia ${ }^{53,56}$. Further, we found that miR-143 expression significantly correlates with the survival of AML patients and is associated with good prognostic factors. These data show high miR-143 expression as a favorable prognostic factor in AML and substantiate a general role for miR-143 in prognosis, which is supported by data in solid cancers and from Elhamamsy et al. in $\mathrm{AML}^{57-59}$. Cancer patient prognosis is strongly affected by the response to chemotherapy. Several studies have suggested miRNAs as novel players in the development of chemotherapy resistance $e^{60-62}$. In this context, low miR-143 levels could be shown to be associated with chemotherapy resistance in solid cancers $^{63,64}$, whereas, miR-143 overexpressing cells show enhanced sensitivity to chemotherapy in these cells ${ }^{65-67}$. In our study, we could show that treatment of AML cells, carrying an FLT3-ITD mutation, with phase III drug AC220 leads to the induction of miR-143 expression whereas stable miR-143 knockdown results in a diminished response to the treatment. Further, we could demonstrate that AML patients expressing high mir-143 levels show better response to 5-azacytidine-based chemotherapy. These data strongly support a role of miR-143 in the chemotherapy response of AML cells. 


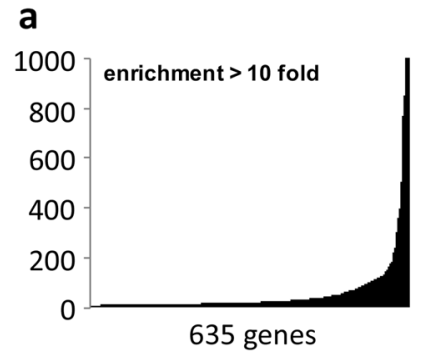

635 genes
MAPK signaling pathway

mTOR signaling pathway

pathways in cancer

MicroRNAs in cancer

p53 signaling pathway

signaling pathways

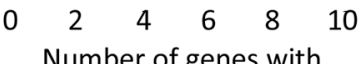

elative enrichment in AGO2

binding $>10$ fold $(n=635)$

b
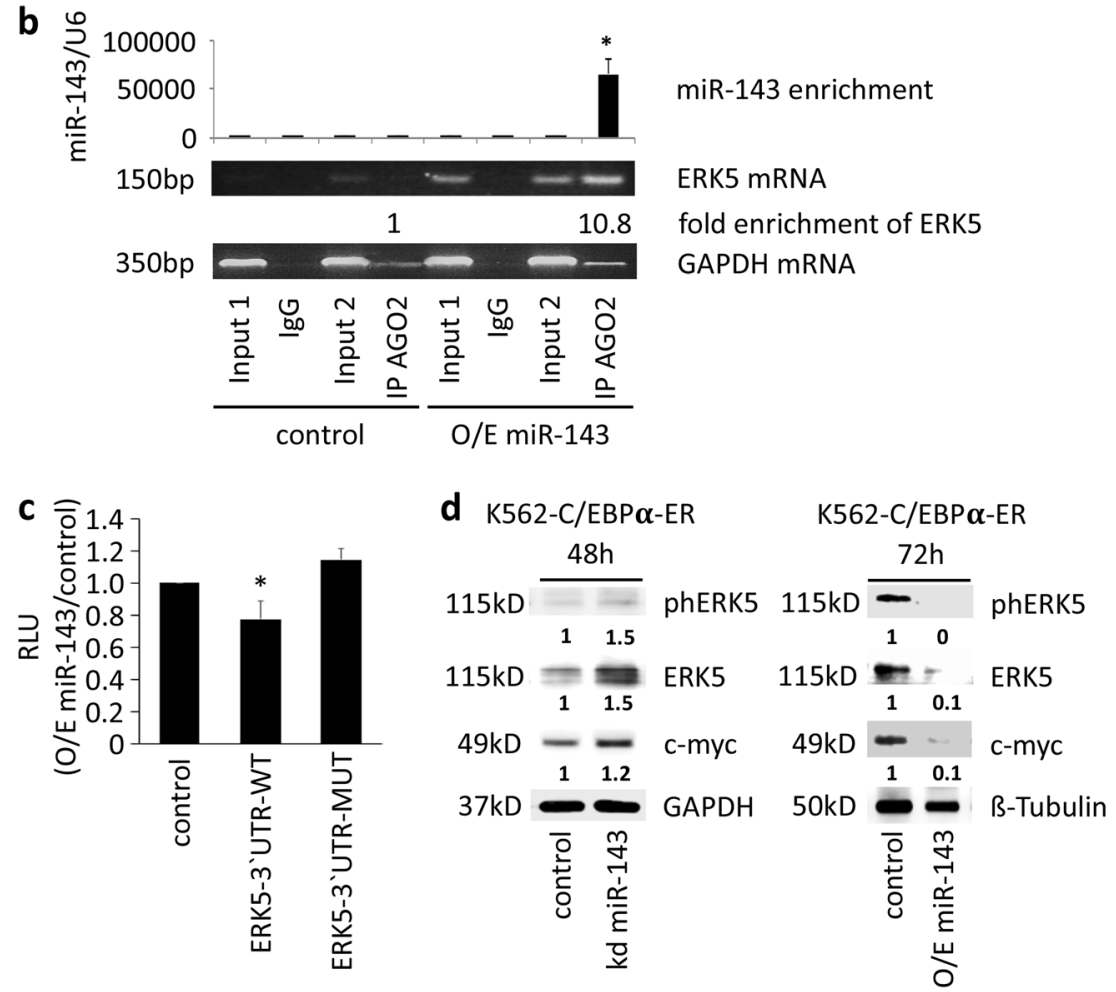

Fig. 6 miR-143 induces ERK5 downregulation. a Enriched genes in miR-143 overexpressing K562-C/EBPa-ER cells after AGO-RIP. Genes detected by RIP-sequencing were plotted against their relative enrichment in AGO2 binding. A 10-fold enrichment in RIP samples was chosen as cut-off for identifying AGO2-bound mRNAs (left). KEGG pathway analysis of genes enriched after AGO-RIP (right). Data represent three independent experiments. b miR-143 and ERK5 mRNA enrichment in AGO-RIP samples. qPCR for miR-143 (upper panel). Bars of the diagram show the fold change of miR-143 expression compared to the input of the control infected cells. Values represent the mean \pm SD from three independent experiments. $P$ values are relative to control. ${ }^{*} P<0.05$; unpaired two-tailed $t$-tests. Semiquantitative PCR for ERK5 mRNA and GAPDH as non-binding control in the AGO-RIP samples (lower panels). The gel pictures represent one example of three independent experiments. Numbers below the gel picture indicate the enrichment of ERK5 mRNA in the AGO-RIP of the miR-143 O/E sample compared to the AGO-RIP of the control sample. $\mathbf{c}$ Luciferase assay for direct miR-143 binding to the $3^{\prime}$ UTR of ERK5. K562-C/EBPa-ER cells were cotransfected using $0.1 \mu \mathrm{g} \mathrm{pRL,} 1 \mu \mathrm{g}$ of either pGL3-ERK5 3' UTR-WT, pGL3ERK5 3'UTR-MUT, or PGL3-C/EBPa 3' UTR in combination with miR-143 or control mimics. Bars represent the luciferase activity for the corresponding vectors. Normalization was done by Renilla luciferase ( $p R L)$. Bars in the diagram represent the mean of 3 independent experiments $\pm S D$. ${ }^{*} P<0.05$; unpaired two-tailed t-tests. $\mathbf{d}$ Western blot for phosphorylated ERK5, ERK5, and c-Myc protein in K562-C/EBPa-ER cells after transient knockdown (left) and overexpression of miR-143 (right) at indicated time points. GAPDH and $\beta$-tubulin were used as control. The western blot pictures represent one example of three independent experiments. Numbers below the blots represent the ratio to the respective controls

In our study, we identified miR-143 mRNA target pathways in myeloid cells by AGO-RIP-Seq. By in silico analysis, we could match several identified genes to basic cellular pathways. Beside pathways involving catalytic activity or nucleic acid and protein binding, members of the MAPK and mTOR pathway were notably highly enriched. Interestingly, the MEK/MAPK pathway is constitutively activated in the majority of AML subtypes and 


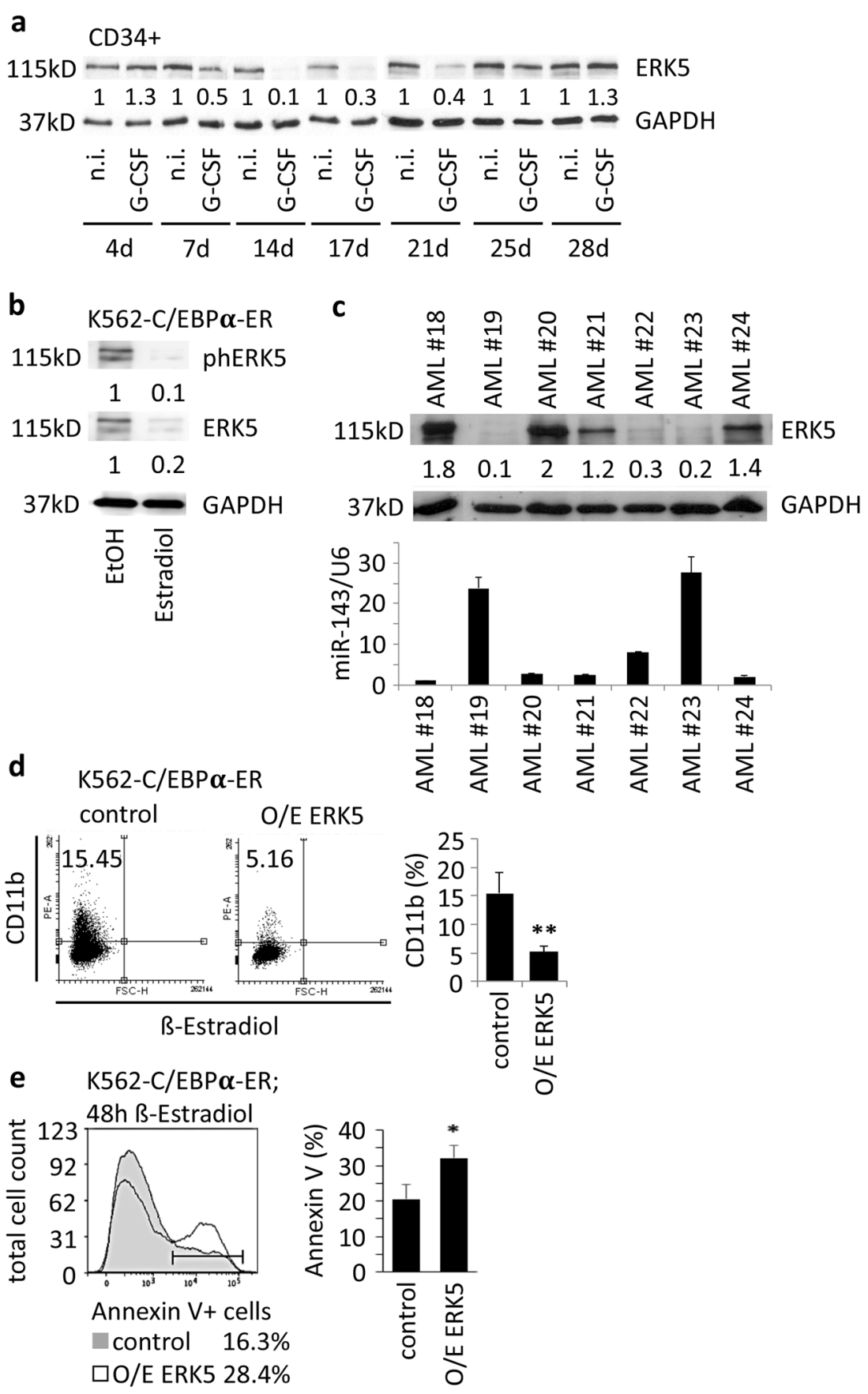

Fig. 7 ERK5 protein inversely correlates with miR-143 expression, interferes with granulocytic differentiation, and is relevant for myeloid cell survival. a Western blot for ERK5 protein in G-CSF-treated CD34 ${ }^{+}$HSPCs at indicated time points. The western blot pictures represent one example of three independent experiments. Numbers below the blots represent the mean ratio to the respective controls. $\mathbf{b}$ Western blot for phosphorylated ERK5 and ERK5 protein in K562-C/EBPa-ER cells $48 \mathrm{~h}$ after $\beta$-estradiol treatment. The western blot pictures represent one example of three independent experiments. Numbers below the blots represent the ratio to the respective controls. c Western blot for ERK5 protein (upper panel) and qPCR for miR-143 expression (lower panel) in primary AML patient samples. GAPDH was used for normalization. The western blot pictures represent one example of three technical replicates. For AML patient samples the ratios were divided by the mean of all samples. The bars in the diagram (lower panel) summarize the mean \pm SD of three technical replicates. $\mathbf{d}$ Transient overexpression of ERK5 in K562-C/EBPa-ER followed by $\beta$ estradiol treatment. FACS analysis for CD11b $48 \mathrm{~h}$ after transfection. Numbers in dot blots indicate percentage of CD11 b+ cells. The bars in the diagram summarize the mean $\pm \mathrm{SD}$ of three independent experiments. $P$ values are relative to control. ${ }^{*} P \leq 0.01$, unpaired two-tailed $t$-tests. e FACS analysis of AnnexinV-stained K562-C/EBPa-ER cells overexpressing ERK5 or control. The bars represent the mean \pm SD of three independent experiments. $P$ values are relative to control. ${ }^{*} P<0.05$, unpaired two-tailed $t$-tests 
confers to uniformly poor prognosis ${ }^{68,69}$. In particular, ERK5, a member of the MAPK-family, was found to be enriched in the AGO-RIP-Seq. Besides its role in cell survival and proliferation, ERK5 is essential for growth of leukemic cells in vivo and one of the key players in chemotherapy resistance, including $\mathrm{AML}^{34,37,38,70-72}$. In the present study, we could identify ERK5 as direct target of miR-143 in hematopoietic cells, which is supported by previous publications showing ERK5 as direct target of miR-143 in solid cancers ${ }^{30,66,73}$. Moreover, the inverse correlation of miR-143 expression and ERK5 protein during granulopoiesis and in AML patient samples substantiate a functional interplay of miR-143 and ERK5 during normal granulocytic differentiation and in AML. Additionally, we could confirm functional involvement of ERK5 in myeloid differentiation ${ }^{32,74}$. These findings are in line with data from Wang et al. suggesting that ERK5 activity directs hematopoietic lineage selection towards the monocytic phenotype as well as with data from Carvajal-Vergara et al. showing that inhibition of ERK5 sensitizes cells to apoptosis ${ }^{74,75}$.

In summary, we show that miR-143 is an important regulator in myeloid differentiation by targeting ERK5. Along with these findings, we show that AML patients with high miR-143 expression show a better OS and benefit from AML treatment strategies (Fig. 8). It seems reasonable to suggest inhibition of the MAPK/MEK signaling by a miR-143 restoration approach as a novel therapeutic approach in AML therapy particularly with regard to chemotherapy resistance in $\mathrm{AML}^{76-78}$. Finally, miR-143 expression may function as a marker for creating

Proliferation
FiR-143
Fig. $\mathbf{8}$ Schematic representation of a model for the role of miR-
$\mathbf{1 4 3}$ in granulopoiesis and in AML. During granulopoiesis, miR-143
expression is upregulated which in turn leads to repression of ERK5
and its downstream target c-myc resulting in myeloid differentiation.
In AML, miR-143 is differentially expressed by various mechanisms.
High miR-143 expression leads to better overall survival of AML
patients whereas low miR-143 AML patients have a poor outcome.
Overexpression of miR-143 in AML cells results in enhanced apoptosis
and alters the susceptibility of those cells to chemotherapy

personalized treatment strategies for patients suffering from this disease.

\section{Materials and methods \\ Primary human samples}

Patient samples were collected with written informed consent in accordance to the Declaration of Helsinki and under Ethics committee approval of each participating institution. AML patient samples were obtained as RNA or frozen bone marrow samples from the OSHO (East German Study Group Hematology/Oncology) patient sample collection at Leipzig University Hospital (Leipzig, Germany) and from Halle University Hospital (Halle, Germany). Blood cell samples from patients without hematopoietic diseases were obtained from the Halle University Hospital. All samples were karyotyped and molecular genetic analysis was performed. Hematopoietic CD34 ${ }^{+}$HSPCs cells were isolated from umbilical cord blood using CD $34^{+}$MicroBead kit (Miltenyi Biotec), as previously described ${ }^{10}$.

\section{Cells, cell culture, treatment, and transfections}

NB4, U937, and 32D-FLT3-ITD cells were grown in RPMI-1640 medium (Life Technologies, Carlsbad, CA, USA) supplemented with $10 \%$ FBS and $1 \%$ penicillin/ streptomycin/glutamine (P/S/G) (Life Technologies). K562C/EBP $\alpha$-ER were grown in RPMI-1640 medium supplemented with $10 \%$ charcoal-treated FBS (PAN-Biotech, Aidenbach, Germany), $1 \%$ P/S/G, and $1 \mu \mathrm{g} / \mathrm{mL}$ puromycin (Merck KGaA, Darmstadt, Germany). 293TN cells were grown in DMEM (Life Technologies) supplemented with $10 \%$ FBS. Primary human CD34 ${ }^{+}$HSPC cells were grown for 3 days in IMDM (Life Technologies) supplemented with $10 \%$ FBS, $50 \mathrm{ng} / \mu \mathrm{L}$ IL-6, SCF and FLT3-L. From day 4 on CD34 ${ }^{+}$HSPCs were grown in IMDM supplemented with $10 \%$ FBS, $10 \mathrm{ng} / \mathrm{mL}$ IL-3, $25 \mathrm{ng} / \mathrm{mL}$ SCF and FLT3-L. Granulocytic differentiation was induced with $100 \mathrm{ng} / \mu \mathrm{L}$ G-CSF starting from day 0 and every second day thereafter. All cytokines were purchased from Immunotools (ImmunoTools $\mathrm{GmbH}$, Friesoythe, Germany). For differentiation of NB4 and U937, cells were induced with $1 \mu \mathrm{M}$ ATRA (Merck KGaA) and DMSO as control. For differentiation of locked nucleic acid (LNA) transfected cells, $0.1 \mu \mathrm{M}$ ATRA was used. Differentiation of K562-C/EBP $\alpha$-ER cells was induced with $1 \mu \mathrm{M} \beta$-estradiol (Merck $\mathrm{KGaA}$ ) and $\mathrm{EtOH}$ as control. 32D-FLT3-ITD cells were treated with $1 \mathrm{nM}$ AC220 (Quizartinib) to inhibit FLT3 signaling.

Transfection of pcDNA6.2 plasmids, pcDNA3.1 plasmids, and LNAs was performed by electroporation (Amaxa Technology, Lonza) using cell specific Nucleofactor-Kits, according to the manufacturer's instructions. For Luciferase assay lipofectamine LTX (ThermoFisher Scientific) was used according to the manufacturer's instructions. Transfection efficiency was measured using 
flow cytometry (FACS) for fluorescent markers and was higher by $50 \%$ in all cells.

\section{miRNA expression profiling}

In total, $500 \mathrm{ng}$ RNA was extracted using TRIzol (ThermoFisher Scientific) method and used for small RNA library preparation with the TruSeqTM Small RNA Sample Prep Kit v2 (Illumina), according to the manufacturer's instructions. The barcoded libraries were size restricted between 140 and $165 \mathrm{bp}$ purified, and quantified using the Library Quantification Kit-Illumina/Universal (KAPA Biosystems), according to the manufacturer's instructions. A pool of up to 12 libraries was used for cluster generation per lane. Library DNA at a concentration of $10 \mathrm{pM}$ was clustered using an Illumina cBot, according to the SR_Amp_Lin_Block_Hybv8.0 protocol of the manufacturer. Sequencing of $50 \mathrm{bp}$ was performed with an Illumina HighScan-SQ sequencer using version 3 chemistry and the version 3 flow cell according to the instructions of the manufacturer. Results were aligned to miRBase v21 and normalized. Median expression of each miRNA was set as threshold for low or high expression. Each experimental group was then analyzed for its level of expression compared to the calculated threshold.

miRNA and mRNA detection by quantitative real-time PCR

Total RNA from cells was extracted using TRIzol. Briefly, cells were spun down and resuspended in $1 \mathrm{~mL}$ TRIzol and extracted with trichloromethane. RNA was then precipitated with isopropanol, washed with EtOH, and air dried. After resuspension of total RNA in water, the amount of RNA was measured using NanoDrop lite Spectrophotometer. miRNA and mRNA reverse transcription was done using $100 \mathrm{ng}$ of RNA and MultiScribe $^{\mathrm{TM}}$ Reverse Transcriptase (ThermoFisher, \#4311235 according to the manufacturer's instructions. Reverse transcription of mRNA was performed using a 50:50 mixture of oligo $\mathrm{dT}$ and random hexamer primers. miRNA reverse transcription was performed using genespecific primers according to the manufacturer's instructions (Applied Biosystems). cDNA samples were used for qPCR analysis in a 1:10 dilution with genespecific primers. qPCR reactions were performed on a MyiQ2 Two-Color Real-Time PCR Detection System (BIORAD) or an Applied Biosystems 7500 Real-Time PCR System (ThermoFisher) using SYBR green for mRNA samples and TaqMan probes for miRNA samples. RNU6B and RNU44 were used for normalization of human miRNAs, snoRNA-135 was used for normalization of mouse miRNAs, and GAPDH was used for normalization of mRNAs. All miRNA primers were obtained from Life Technologies. Specific mRNA primers were obtained from Biomers. Primer sequences for ERK5 and GAPDH are provided in Supplementary Table 7. Data analysis was done using $2-{ }^{\Delta \Delta \mathrm{Ct}}$ method.

\section{DNA constructs and cloning}

The pcDNA6.2 expression vector and the pcDNA6.2 control vector, used for transient overexpression of miR143 or control, were purchased from ThermoFisher Scientific. The pre-miR-143 sequence was cloned into the pcDNA6.2 expression vector according to the manufactures instructions (Supplementary Table 7). The pcDNA3.1-ERK5 was a kind gift from A. Martin-Villalba (IRB, Montpellier). The pcDNA3.1 control was obtained from ThermoFisher Scientific.

\section{Virus production and cell infection}

Lentiviral vector (pLV-miR-143) for overexpression of miR-143 or an unspecific control (pLV-control) sequence was purchased from Biosettia (Biosettia Inc., San Diego, CA, USA). Both of them contained RFP reporter and showed puromycin resistance. Lentiviral vector for knockdown of miR-143 (miRZip-143) and the corresponding unspecific control (miRZip-control) vector were purchased from System Biosciences (System Biosciences, LLC, Palo Alto, CA, USA). Both of them contained GFP reporter and showed puromycin resistance. Pseudoviral particles were produced according to the manufacturer's instructions using psPAX2, pMD2G in 293TN cells. Target cells were infected for $24 \mathrm{~h}$ with PEG-concentrated (System Biosciences) viral particles followed by puromycin selection or FACS sorting. Purity of transduced cells was analyzed using flow cytometry for expression of fluorescent markers and was $>90 \%, 7$ days after selection or FACS sorting. Stable transduced cells were maintained in media supplemented with $1 \mu \mathrm{g} / \mathrm{mL}$ puromycin.

\section{Isolation of bone marrow precursor populations and granulocytes}

Distinct murine bone marrow populations were sorted from C57BL/6NCrl mice, as previously described ${ }^{10}$.

\section{LSK cell isolation, manipulation, and transplantation into mice}

Purification and culture of lineage ${ }^{-} \mathrm{Sca}-1^{+} \mathrm{cKit}^{+}$(LSK) cells was done as previously described ${ }^{9,79}$. Briefly, LSK cells were sorted from BM isolated from hips, legs, and spine of 12-17-week-old male C57Bl/6NCrl $\left(\right.$ Ly5.2 $\left.2^{+}\right)$ mice. Sorted LSK cells were plated into StemSpan SFEM medium (Stemcell Technologies, Vancouver, Canada) supplemented with $10 \mathrm{ng} / \mathrm{mL} \mathrm{mIL}-3,20 \mathrm{ng} / \mathrm{mL}$ hIL-6, $100 \mathrm{ng} / \mathrm{mL} \mathrm{mSCF}, 50 \mathrm{ng} / \mathrm{mL} \mathrm{mTPO}, 100 \mathrm{ng} / \mathrm{mL} \mathrm{mFlt}-3$ ligand (all from PeproTech, Rocky Hill, NJ, USA), and $8 \mu \mathrm{g} / \mathrm{ml}$ polybrene (Sigma). LSK cells were then transduced with pseudoviral particles carrying miRZip-143 or 
miRZip-control lentivector (both containing GFP reporter). Virus was added at MOI of 20 for $6 \mathrm{~h}$. Next, cells were washed twice with PBS, cultured for another $18 \mathrm{~h}$, and then transplanted into recipient mice by tail vein injection. Congenic C57BL/6NCrl mice $\left(\mathrm{Ly} 5.1^{+}\right.$) were employed as recipients and they were lethally irradiated (two doses of $7.5 \mathrm{~Gy}$, separated by $4 \mathrm{~h}$ ). $5 \times 10^{4}$ transduced LSK cells $\left(\right.$ Ly5.2 $2^{+}$) were mixed with $5 \times 10^{5}$ whole bone marrow cells as a support $\left(\right.$ Ly $\left.5.1^{+}\right)$. Forty-eight hour after transduction, a non-transplanted aliquot of transduced cells was analyzed by flow cytometry for GFP positivity to assess efficiency of transduction. Recipient mice were bled and killed 3 weeks after transplantation and bone marrow was isolated. All mice that showed no proper engraftment $\left(\right.$ Ly5. $\left.2^{+}<0.5 \%\right)$, did not express GFP, or showed general abnormalities in the $\mathrm{GFP}^{-}$fraction were removed from final consideration. All mice experiments were approved by the Animal Care and Use Committee of the Institute of Molecular Genetics and were in agreement with local legal requirements and ethical guidelines.

\section{May-Grünwald/Giemsa staining, morphological analysis, and BM differential counting}

For cytospin analysis, $5 \times 10^{4}-1 \times 10^{5}$ cells were spun down on glass slides and stained with May-Grünwald and Giemsa staining solution (Dr. K. Hollborn \& Söhne, Leipzig, Germany) according to the manufacturer's instructions. Morphological analysis and manual leukocyte differential counts of bone marrow cells were performed using May-Grünwald/Giemsa stained cytospins. Cytospins were prepared from sorted bone marrow Ly5.2 $2^{+} \mathrm{GFP}^{+}$ cells from mice transplanted with LSK cells transduced with control or miR143 knockdown constructs. A minimum of 100 cells per cytospin was analyzed.

\section{Flow cytometry and fluorescence-activated cell sorting}

For flow cytometry analysis, cells were prepared according to the manufacturer's instructions and stained with specific antibodies. Labeled cells were analyzed on a BD LSR II or BD ARIA cytometer using CellQuest software (BD Biosciences). Final evaluation of data was done using FCS Express v6 (De Novo Software, Glendale, CA or FlowJo, FlowJo LLC).

Sorting of $\mathrm{GFP}^{+}$cells and murine bone marrow subpopulations was performed as previously described ${ }^{8}$. For measurement of apotosis Annexin V Apoptosis Detection Kit I was used according to manufacturer's instructions (BD Pharmingen \#556547, \#559763). All antibodies used for flow cytometry are listed in Supplementary Table 8.

\section{Cell growth assay}

32D-FLT3-ITD cells stably transduced with pLV-miR143 or control vector were plated in a density of $1 \times 10^{4}$ cells $/ \mathrm{mL}$. Proliferation rate was ascertained by cell counting in a Neubauer chamber using Trypan blue staining for excluding dead cells.

\section{Argonaute2-RNA-immunoprecipitation (AGO-RIP) and next-generation sequencing (NGS)}

RNA-immunoprecipitation was performed on $\mathrm{K} 562-\mathrm{C} /$ EBP $\alpha$-ER cells stably overexpressing miR-143 or control using Imprint ${ }^{\oplus}$ RNA Immunoprecipitation Kit (Sigma Aldrich), according to the manufacturer's instruction. For each individual sample $3 \times 10^{6}$ cells were used. $500 \mathrm{ng}$ total RNA from AGO-RIP sample preparation was extracted using TRIzol. RNA library preparation was done with the TruSeq RNA Library Preparation Kit v2 (Illumina) according to the manufacturer's instructions. Results were processed using TopHat and Cufflinks according to John L Rinn \& Lior Pachter et al. ${ }^{80}$

\section{Luciferase assay}

Luciferase assay constructs carrying the wild-type ERK5-3' UTR and the ERK5-3' UTR with the mutated miR-143 binding site between the luciferase ORF and the poly A tail were a kind gift from Prof. L. Fajas (Center for Integrative Genomics, CIG, Lausanne, Switzerland ${ }^{30}$. The corresponding pGL3-control vector was purchased from Promega (Promega GmbH, Mannheim, Germany). Luciferase assays were performed in K562-C/EBP $\alpha$-ER cells by co-transfection of the respective pGL3 construct, pRL (Renilla construct, and 200nmol miR-143 mimic molecules or negative control mimics (Dharmacon, Lafayette, CO, USA) using Lipofectamine LTX reagent. Luciferase activity was measured using the Dual Luciferase Reporter Assay (Promega) 24h after transfection according to the manufacturer's instructions. Cotransfected pRL luminescence was used for normalization of luciferase activity.

\section{Western blot}

Western blots were performed as previously described (9). Briefly, cells were harvested in standard RIPA buffer supplemented with protease and phosphatase inhibitor cocktails (Roche). The amount of protein was measured using Protein Assay Dye Reagent Concentrate (BIORAD) according to the manufacturer's instructions using a Nanophotometer (Implen). Equal amounts of protein was loaded on a PAGE. Proteins were blotted semi dry with a Trans-Blot Turbo (BIORAD) according to the manufacturer's instructions. Membranes were blocked with 5\% BSA for $1 \mathrm{~h}$ at room temperature. If possible, the western blot membrane was cut to simultaneously probe for low- and high-molecular weight protein with 1 st antibody at $4{ }^{\circ} \mathrm{C}$ overnight. Membranes were then washed probed with 2nd antibody and washed again. Probing of membranes with two different antibodies of the same size was done by stripping of the 
membrane and re-probing with antibody following the aforementioned procedure. Immunodetection was performed using WesternSure Blot Ultra- or WesternSure Blot Premium Substrate (Licor, Lincoln, Nebraska). Membranes were analyzed using a C-Digit Chemiluminescent Western Blot Scanner (Licor). Evaluation of data was performed using ImageJ software (NIH, Bethesda, MD, USA). All antibodies used for western blot are listed in Supplementary Table 8.

\section{Statistical analyses}

All experiments were performed at least three times except for primary AML samples where at least two independent reverse transcription reactions and corresponding qPCRs were performed. Data are presented as means \pm standard deviation. Statistical analyses were performed using SPSS (IBM, New York, USA) or Excel (Microsoft Corporation, Redmond, WA, USA). Student $t$-, Mann-Whitney $U$-, or log rank tests were performed to determine statistical significance of experimental results. A $p$-value of $P \leq 0.05$ was considered significant $(*)$ and a $p$-value of $\leq 0.01{ }^{(* *)}$ ) was considered as highly significant.

\section{Accession numbers}

Data from next-generation sequencing have been deposited in the Gene Expression Omnibus (GEO) with the accession numbers GSE93549 and GSE93550.

\section{Acknowledgements \\ The authors thank Prof. Dr. Liebert and Dr. Brinckmann from the Institute of Virology, University of Leipzig, for helping in establishing the production of lentiviral particles and Dr. Michael Cross from the Division of Hematology and Oncology, University of Leipzig, for providing umbilical cord blood cells for the cell culture experiments. The authors also thank PD Dr. Knut Krohn from the Core Facility DNA sequencing of the Leipzig University for performing the small RNA and mRNA deep sequencing procedure and helping with the analysis. This study was supported by grants from the DFG - German Research Foundation (BE 2042/12-1; GRK1591), Deutsche José Carreras Leukämie- Stiftung e.V. (DJCLS R 11/17 and DJCLS R 15/18 to GB), Zusammen gegen den Krebs e.V., and Wilhelm Sander Stiftung (no. 2013.153.1 and no. 2015.093.1) to $\mathrm{GB}$, and support from the institute of Molecular Genetics of the Czech Academy of Sciences (RVO 68378050) to MAJ.}

\section{Author details}

'Division of Hematology and Oncology, Leipzig University Hospital, Leipzig, Germany. ${ }^{2}$ Institute of Molecular Genetics of the ASCR, v.v.i. Laboratory of Haematooncology, Prague, Czech Republic. ${ }^{3}$ Department of Internal Medicine V, University of Heidelberg, Heidelberg, Germany

\section{Conflict of interest}

The authors declare that they have no conflict of interest.

\section{Publisher's note}

Springer Nature remains neutral with regard to jurisdictional claims in published maps and institutional affiliations.

Supplementary Information accompanies this paper at (https://doi.org/ 10.1038/s41419-018-0837-x).
Received: 20 December 2017 Revised: 30 May 2018 Accepted: 26 June 2018

Published online: 26 July 2018

\section{References}

1. Bartel, D. P. MicroRNAs: target recognition and regulatory functions. Cell 136 215-233 (2009).

2. Lee, R. C., Feinbaum, R. L. \& Ambros, V. The C. elegans heterochronic gene lin4 encodes small RNAs with antisense complementarity to lin-14. Cell 75, 843-854 (1993).

3. Brennecke, J. \& Cohen, S. M. Towards a complete description of the microRNA complement of animal genomes. Genome Biol. 4, 228 (2003).

4. Hatfield, S. D. et al. Stem cell division is regulated by the microRNA pathway. Nature 435, 974-978 (2005).

5. Guo, S. et al. MicroRNA miR-125a controls hematopoietic stem cell number. Proc. Natl. Acad. Sci. USA 107, 14229-14234 (2010).

6. Fazi, F. et al. A minicircuitry comprised of microRNA-223 and transcription factors NFI-A and C/EBPalpha regulates human granulopoiesis. Cell 123, 819-831 (2005).

7. Bräuer-Hartmann, D. et al. PML/RARa-regulated miR-181a/b cluster targets the tumor suppressor RASSF1A in acute promyelocytic leukemia. Cancer Res. 75, 3411-3424 (2015).

8. Gerloff, D. et al. NF-kB/STAT5/miR-155 network targets PU.1 in FLT3-ITD-driven acute myeloid leukemia. Leukemia 29, 535-547 (2015).

9. Wurm, A. A. et al. Disruption of the C/EBPa-miR-182 balance impairs granulocytic differentiation. Nat. Commun. 8, 46 (2017).

10. Katzerke, C. et al. Transcription factor C/EBPa-induced microRNA-30c inactivates Notch1 during granulopoiesis and is downregulated in acute myeloid leukemia. Blood 122, 2433-2442 (2013).

11. Pulikkan, J. A. et al. C/EBPa regulated microRNA-34a targets E2F3 during granulopoiesis and is down-regulated in AML with CEBPA mutations. Blood 116, 5638-5649 (2010).

12. Stamato, M. A. et al. Inhibition of EZH2 triggers the tumor suppressive miR-29b network in multiple myeloma. Oncotarget 8, 106527-106537 (2017).

13. Ferrara, F. \& Schiffer, C. A. Acute myeloid leukaemia in adults. Lancet (Lond., Engl.) 381, 484-495 (2013).

14. Marcucci, G., Haferlach, T. \& Döhner, H. Molecular genetics of adult acute myeloid leukemia: prognostic and therapeutic implications. J. Clin. Oncol. 29, 475-486 (2011).

15. Marcucci, G., Mrózek, K., Radmacher, M. D., Garzon, R. \& Bloomfield, C. D. The prognostic and functional role of microRNAs in acute myeloid leukemia. Blood 117, 1121-1129 (2011).

16. Chen, $X$. et al. Clinical value of integrated-signature miRNAs in colorectal cancer: miRNA expression profiling analysis and experimental validation. Oncotarget 6, 37544-37556 (2015).

17. Votavova, H. et al. Differential expression of microRNAs in CD34 + cells of 5qsyndrome. J. Hematol. Oncol. 4, 1 (2011).

18. lio, A., Nakagawa, Y., Hirata, l., Naoe, T. \& Akao, Y. Identification of non-coding RNAs embracing microRNA-143/145 cluster. Mol. Cancer 9, 136 (2010).

19. Cordes, K. R. et al. miR-145 and miR-143 regulate smooth muscle cell fate and plasticity. Nature 460, 705-710 (2009).

20. Zhang, H.P. et al. A regulatory circuit involving miR-143 and DNMT3a mediates vascular smooth muscle cell proliferation induced by homocysteine. Mol. Med. Rep. 13, 483-490 (2016).

21. Jin, Y.-P. et al. miR-143-3p targeting of ITGA6 suppresses tumour growth and angiogenesis by downregulating PLGF expression via the PI3K/AKT pathway in gallbladder carcinoma. Cell Death Dis. 9, 182 (2018).

22. Guoping M., Ran L., Yanru Q. miR-143 inhibits cell proliferation of gastric cancer cells through targeting GATA6. Oncol Res. https://doi.org/10.3727/ $096504018 \times 15151515028670$ (2018).

23. Zhang, Q., Feng, Y., Liu, P. \& Yang, J. MiR-143 inhibits cell proliferation and invasion by targeting DNMT3A in gastric cancer. Tumour Biol. 39, 1010428317711312 (2017).

24. Kent, O. A. et al. Repression of the miR-143/145 cluster by oncogenic Ras initiates a tumor-promoting feed-forward pathway. Genes Dev. 24, 2754-2759 (2010).

25. Lei, C. et al. miR-143 and miR-145 inhibit gastric cancer cell migration and metastasis by suppressing MYO6. Cell Death Dis. 8, e3101 (2017). 
26. Piatopoulou, D. et al. Clinical utility of miR-143/miR-182 levels in prognosis and risk stratification specificity of BFM-treated childhood acute lymphoblastic leukemia. Ann. Hematol. . https://doi.org/10.1007/s00277-018-3292-y (2018).

27. Drew, B. A., Burow, M. E. \& Beckman, B. S. MEK5/ERK5 pathway: the first fifteen years. Biochim Biophys. Acta-Rev. Cancer 1825, 37-48 (2012).

28. Xia, C., Yang, Y., Kong, F., Kong, Q. \& Shan, C. MiR-143-3p inhibits the proliferation, cell migration and invasion of human breast cancer cells by modulating the expression of MAPK7. Biochimie 147, 98-104 (2018).

29. Dong, $\mathbf{X}$. et al. MiR-143 regulates the proliferation and migration of osteosarcoma cells through targeting MAPK7. Arch. Biochem. Biophys. 630, 47-53 (2017).

30. Clapé, C. et al. miR-143 interferes with ERK5 signaling, and abrogates prostate cancer progression in mice. PLoS One 4, e7542 (2009).

31. Williams, C. A. C. et al. Erk5 is a key regulator of naive-primed transition and embryonic stem cell identity. Cell Rep. 16, 1820-1828 (2016).

32. Angulo-lbáñez, M. et al. Erk5 contributes to maintaining the balance of cellular nucleotide levels and erythropoiesis. Cell Cycle 14, 3864-3876 (2015).

33. Nithianandarajah-Jones, G. N., Wilm, B., Goldring, C. E. P., Müller, J. \& Cross, M. J. ERK5: structure, regulation and function. Cell Signal. 24, 2187-2196 (2012).

34. Giurisato, E. et al. Myeloid ERK5 deficiency suppresses tumor growth by blocking protumor macrophage polarization via STAT3 inhibition. Proc. Natl. Acad. Sci. USA 115, E2801-E2810 (2018).

35. Tusa, I. et al. ERK5 is activated by oncogenic BRAF and promotes melanoma growth. Oncogene 37, 2601-2614 (2018).

36. Granados-Jaén, A. et al. Absence of ERK5/MAPK7 delays tumorigenesis in Atm-/- mice. Oncotarget 7, 74435-74447 (2016).

37. Simões, A. E. S., Rodrigues, C. M. P. \& Borralho, P. M. The MEK5/ERK5 signalling pathway in cancer: a promising novel therapeutic target. Drug Discov. Today 21, 1654-1663 (2016).

38. Xu, Y., Cao, C., Gong, X. \& Rong, L. Inhibition of ERK5 enhances cytarabineinduced apoptosis in acute myeloid leukemia cells. Int. J. Clin. Exp. Med. 8, 6446-6455 (2015)

39. Levis, M. \& Small, D. FLT3: ITDoes matter in leukemia. Leuk. J. Leuk. Soc. Am. Leuk. Res Fund., UK 17, 1738-1752 (2003).

40. Croce, C. M. \& Calin, G. A. miRNAs, cancer, and stem cell division. Cell 122, 6-7 (2005).

41. Zhang, B., Pan, X., Cobb, G. P. \& Anderson, T. A. microRNAs as oncogenes and tumor suppressors. Dev. Biol. 302, 1-12 (2007).

42. Chen, L. et al. MicroRNA-143 regulates adipogenesis by modulating the MAP2K5-ERK5 signaling. Sci. Rep. 4, 3819 (2014).

43. Batliner, J., Buehrer, E., Fey, M. F. \& Tschan, M. P. Inhibition of the miR-143/145 cluster attenuated neutrophil differentiation of APL cells. Leuk. Res. 36, 237-240 (2012).

44. Ng, E. K. O. et al. microRNA-143 is downregulated in breast cancer and regulates DNA methyltransferases 3A in breast cancer cells. Tumour Biol. 35, 2591-2598 (2014)

45. Zhou, P., Chen, W.-G. \& Li, X.-W. microRNA-143 acts as a tumor suppressor by targeting hexokinase 2 in human prostate cancer. Am. J. Cancer Res. 5 2056-2063 (2015)

46. Wei, J. et al. miR-143 inhibits cell proliferation by targeting autophagy-related 2B in non-small cell lung cancer H1299 cells. Mol. Med. Rep. 11, 571-576 (2015).

47. Xia, H. et al. miR-143 inhibits NSCLC cell growth and metastasis by targeting Limk1. Int. J. Mol. Sci. 15, 11973-11983 (2014).

48. Zhang, Y. et al. microRNA-143 targets MACC1 to inhibit cell invasion and migration in colorectal cancer. Mol. Cancer 11, 23 (2012).

49. Donahue, R. E. et al. Plerixafor (AMD3100) and granulocyte colony-stimulating factor (G-CSF) mobilize different CD34 + cell populations based on global gene and microRNA expression signatures. Blood 114, 2530-2541 (2009).

50. Merkerova, M., Vasikova, A., Belickova, M. \& Bruchova, H. microRNA expression profiles in umbilical cord blood cell lineages. Stem Cells Dev. 19, 17-26 (2010)

51. Jakob, P. \& Landmesser, U. Role of microRNAs in stem/progenitor cells and cardiovascular repair. Cardiovasc. Res. 93, 614-622 (2012).

52. Benati, $M$. et al. Role of JAK2 V617F mutation and aberrant expression of microRNA-143 in myeloproliferative neoplasms. Clin. Chem. Lab. Med. 53, 1005-1011 (2015)

53. Dou, L. et al. Methylation-mediated repression of microRNA-143 enhances MLL-AF4 oncogene expression. Oncogene 31, 507-517 (2012).

54. Volinia, S. et al. Reprogramming of miRNA networks in cancer and leukemia. Genome Res. 20, 589-599 (2010).
55. Shen, J.-Z., Zhang, Y.-Y., Fu, H.-Y., Wu, D.-S. \& Zhou, H.-R. Overexpression of microRNA-143 inhibits growth and induces apoptosis in human leukemia cells. Oncol. Rep. 31, 2035-2042 (2014).

56. Akao, Y., Nakagawa, Y., lio, A. \& Naoe, T. Role of microRNA-143 in Fas-mediated apoptosis in human T-cell leukemia Jurkat cells. Leuk. Res. 33, 1530-1538 (2009).

57. Elhamamsy, A. R. et al. Circulating miR-92a, miR-143 and miR-342 in plasma are novel potential biomarkers for acute myeloid leukemia. Int. J. Mol. Cell Med. 6 77-86 (2017).

58. Guo, H. et al. The regulation of Toll-like receptor 2 by miR-143 suppresses the invasion and migration of a subset of human colorectal carcinoma cells. Mol. Cancer 12, 77 (2013).

59. Avgeris, M. et al. Uncovering the clinical utility of miR-143, miR-145 and miR224 for predicting the survival of bladder cancer patients following treatment. Carcinogenesis 36, 528-537 (2015).

60. Siemens, H., Jackstadt, R., Kaller, M. \& Hermeking, H. Repression of c-Kit by p53 is mediated by miR-34 and is associated with reduced chemoresistance, migration and stemness. Oncotarget 4, 1399-1415 (2013).

61. Xia, L. et al. miR-15b and miR-16 modulate multidrug resistance by targeting BCL2 in human gastric cancer cells. Int. J. Cancer 123, 372-379 (2008).

62. Krakowsky, R. H. E. et al. miR-451a abrogates treatment resistance in FLT3-ITDpositive acute myeloid leukemia. Blood Cancer J. 8, 36 (2018).

63. Boren, $T$. et al. MicroRNAs and their target messenger RNAs associated with ovarian cancer response to chemotherapy. Gynecol. Oncol. 113, 249-255 (2009).

64. Kopczyńska, E. Role of microRNAs in the resistance of prostate cancer to docetaxel and paclitaxel. Współczesna Onkol. 6, 423-427 (2015).

65. Xu, B. et al. miR-143 decreases prostate cancer cells proliferation and migration and enhances their sensitivity to docetaxel through suppression of KRAS. Mol. Cell. Biochem. 350, 207-213 (2011).

66. Wang, L. et al. MiR-143 acts as a tumor suppressor by targeting N-RAS and enhances temozolomide-induced apoptosis in glioma. Oncotarget $\mathbf{5}$ 5416-5427 (2014)

67. Gomes, S. E. et al. Convergence of miR-143 overexpression, oxidative stress and cell death in HCT116 human colon cancer cells. PLoS One 13, e0191607 (2018).

68. Kim, S. C. et al. Constitutive activation of extracellular signal-regulated kinase in human acute leukemias: combined role of activation of MEK, hyperexpression of extracellular signal-regulated kinase, and downregulation of a phosphatase, PAC1. Blood 93, 3893-3899 (1999)

69. Towatari, M. et al. Constitutive activation of mitogen-activated protein kinase pathway in acute leukemia cells. Leukemia 11, 479-484 (1997).

70. Perez-Madrigal, D., Finegan, K. G., Paramo, B. \& Tournier, C. The extracellularregulated protein kinase 5 (ERK5) promotes cell proliferation through the down-regulation of inhibitors of cyclin dependent protein kinases (CDKs). Cell. Signal. 24, 2360-2368 (2012).

71. Garaude, J. et al. ERK5 activates NF-kappaB in leukemic T cells and is essential for their growth in vivo. J. Immunol. 177, 7607-7617 (2006).

72. Zheng, R. \& Studzinski, G. P. Optimal AraC-cytotoxicity to aml cells requires erk5 activity. J. Cell. Biochem. 118, 1583-1589 (2017).

73. Ahmad, I. et al. Mir143 expression inversely correlates with nuclear ERK5 immunoreactivity in clinical prostate cancer. Br. J. Cancer 108, 149-154 (2013).

74. Wang, X., Pesakhov, S., Harrison, J. S., Danilenko, M. \& Studzinski, G. P. ERK5 pathway regulates transcription factors important for monocytic differentiation of human myeloid leukemia cells. J. Cell. Physiol. 229, 856-867 (2014).

75. Carvajal-Vergara, X. et al. Multifunctional role of Erk5 in multiple myeloma. Blood 105, 4492-4499 (2005).

76. Piloto, O. et al. Prolonged exposure to FLT3 inhibitors leads to resistance via activation of parallel signaling pathways. Blood 109, 1643-1652 (2007).

77. McCubrey, J. A. et al. Roles of the Raf/MEK/ERK pathway in cell growth malignant transformation and drug resistance. Biochim Biophys. Acta-Mol. Cell Res. 1773, 1263-1284 (2007)

78. McCubrey, J. A. et al. Ras/Raf/MEK/ERK and PI3K/PTEN/Akt/mTOR cascade inhibitors: how mutations can result in therapy resistance and how to overcome resistance. Oncotarget 3, 1068-1111 (2012).

79. Zjablovskaja, P. et al. EVI2B is a C/EBPa target gene required for granulocytic differentiation and functionality of hematopoietic progenitors. Cell Death Differ. 24, 705-716 (2017).

80. Trapnell, C. et al. Differential gene and transcript expression analysis of RNAseq experiments with TopHat and Cufflinks. Nat. Protoc. 7, 562-578 (2012). 